(c) American Dairy Science Association, 2003.

\title{
Intravaginal Progesterone Insert to Synchronize Return to Estrus of Previously Inseminated Dairy Cows ${ }^{1}$
}

\author{
J. R. Chenault, J. F. Boucher, K. J. Dame, J. A. Meyer, and S. L. Wood-Follis \\ Product Development - Food Animal, Pharmacia Animal Health, Kalamazoo, MI 49001
}

\begin{abstract}
An intravaginal progesterone insert (CIDR insert; $1.38 \mathrm{~g}$ of progesterone) was evaluated for synchronization of returns to estrus (SR), conception rate (CR), and pregnancy rate (PR) in dairy cows previously artificially inseminated (AI). Healthy, nonpregnant, lactating Holstein cows, $\geq 40$ and $\leq 150 \mathrm{~d}$ postpartum at eight commercial farms were used. Cows detected in estrus and receiving AI 2, 3, or $4 \mathrm{~d}$ after one injection of $\mathrm{PGF}_{2 \alpha}(25 \mathrm{mg})$ were assigned as either controls (n $=945)$, or to receive a CIDR insert $(n=948)$ for $7 \mathrm{~d}(14$ to $21 \pm 1 \mathrm{~d}$ after $\mathrm{AI}$ ). Cows were observed for returns to estrus from 18 to $26 \pm 1 \mathrm{~d}$ after initial AI (resynchrony period) and were reinseminated if in estrus. Vaginal mucus on CIDR inserts (97.3\% retention) at removal was scored: $1=$ no mucus; $2=$ clear; $3=$ cloudy; $4=$ yellow; and $5=$ red or brown. Percentage of cows in estrus (SR) during the $3 \mathrm{~d}$ after CIDR insert removal was contrasted to the highest 3 -d cumulative percentage in estrus for controls. Cows conceiving to initial AI were omitted in calculations of SR, CR, and PR during resynchrony. Mucous scores of 3 or 4 (mild irritation) were observed in $65 \%$ of cows and a score of 5 (more severe irritation) was observed in $2 \%$; otherwise, health was unaffected. The PR to initial AI was lower for cows subsequently receiving CIDR inserts than for controls (32.7 vs. $36.7 \%)$. The CIDR insert increased SR ( 34.1 vs. $19.3 \%$ in $3 \mathrm{~d}$ ) and overall estrus detection ( $43 \%$ in $4 \mathrm{~d}$ vs. $36 \%$ in $9 \mathrm{~d}$ ) compared with controls. For the 9-d resynchrony period, CR and PR for CIDR-treated $(26.7,12.2 \%)$ and control (30.9, $11.1 \%$ ) cows did not differ significantly. The CIDR inserts improved synchrony of returns to estrus, slightly reduced PR to initial AI, but did not affect CR or PR to AI during the resynchrony period.
\end{abstract}

Received: August 6, 2002.

Accepted: December 20, 2002.

Corresponding author: J. R. Chenault; e-mail: John.R.Chenault @Pharmacia.com.

${ }^{1}$ Conducted under INAD 6224, FDA, and consistent with FDA Guidance to Industry: Good Target Animal Study Practices for Clinical Investigations (May 1997).
(Key words: intravaginal progesterone intravaginal insert, resynchronization of estrus, dairy cow)

Abbreviation key: CIDR insert = intravaginal insert containing $1.38 \mathrm{~g}$ of progesterone, CIDR B = intravaginal insert containing $1.9 \mathrm{~g}$ of progesterone, $\mathbf{C R}$ = conception rate, $\mathbf{E X D}=$ experimental day, $\mathbf{P R}=$ pregnancy rate, $\mathbf{P} \mathbf{R}_{\text {pre }}=$ pregnancy rate to AI during the preenrollment period, $\mathbf{S R}=$ synchronization rate; percentage of cows in estrus on EXD 25 to 27.

\section{INTRODUCTION}

Numerous programs utilizing $\mathrm{PGF}_{2 \alpha}$ or analogs are available for synchronization of estrus in lactating dairy cows. However, hormonal products are not available in the United States to allow synchronization of the return to estrus in those cattle that have been inseminated but did not conceive to that insemination. Cattle that fail to conceive to an insemination are expected to return to estrus 18 to $24 \mathrm{~d}$ after insemination, the interval of a normal estrous cycle. However, detection of estrus is increasingly challenging for breeding management of dairy herds, particularly in larger herds, among cows in confinement, among cows housed entirely on concrete, and with increasing levels of milk production (Lucy, 2001). Therefore, many cows that do not conceive after AI are not detected in estrus at the first eligible estrus and are not identified as having failed to conceive until diagnosed not pregnant at scheduled pregnancy examinations 35 to 60 $\mathrm{d}$ after insemination. This results in a minimum of a 35-d interbreeding interval and lengthened interval from calving to conception.

Globally, two intravaginal progesterone inserts containing either $1.9 \mathrm{~g}$ (CIDR B inserts) or $1.38 \mathrm{~g}$ of progesterone (CIDR inserts; EAZI-BREED CIDR Cattle Insert) are marketed (Pharmacia Animal Health, Kalamazoo, MI). Plasma progesterone concentrations are indistinguishable over a 14-d interval during the administration of these two products to ovariectomized, nonlactating, Friesian dairy cows (Ogle 1999). Therefore, it is assumed that administration of either product to intact cows or heifers for $7 \mathrm{~d}$ would result in similar efficacy. 
The CIDR B insert has been marketed since the late 1980's and the CIDR insert was introduced in the late 1990's. Therefore, more studies utilizing the CIDR B inserts have been reported in the public domain. New CIDR B inserts (Macmillan and Peterson, 1993; Macmillan and Burke, 1996; Van Cleeff et al., 1996) and previously used CIDR inserts (El-Zarkouny et al., 2001) have been used to synchronize the return to estrus in cattle inseminated at the immediately preceding estrus. Such use also has been referred to as "resynchronization" of estrus. In these studies, inserts were administered between 12 to $17 \mathrm{~d}$ after insemination and removed 20 or $21 \mathrm{~d}$ after insemination (i.e., a 3- to 8-d insertion period). The pregnancy status of cows cannot be determined as early as 12 to $17 \mathrm{~d}$ after insemination. Therefore, all inseminated cows were administered an insert, without knowledge as to their pregnancy status. Cows returned to estrus 1 to $4 \mathrm{~d}$ after removal of the insert, whereas untreated cows returned to estrus over approximately a 7-d interval (18 to $24 \mathrm{~d}$ following insemination). Cows conceiving to the preadministration insemination are not expected to display estrous behavior following insert removal.

The CIDR-B insert has been used in conjunction with an injection of $\mathrm{PGF}_{2 \alpha}$ to synchronize estrus in lactating dairy cows. However, treatment of dairy cows with a CIDR-B insert for $8 \mathrm{~d}$ with an injection of $\mathrm{PGF}_{2 \alpha} 1$ (Ryan et al., 1995) or $2 \mathrm{~d}$ (Xu et al., 1996) before insert removal resulted in a reduced conception rate to AI conducted at the synchronized estrus. This reduced conception rate (CR) is thought to be due to development of persistent follicles in those cows that spontaneously regress their corpus luteum during the insertion period and are exposed only to progesterone released by the insert (Savio et al., 1993a, 1993b; Cooperative Regional Research Project, 1996; Revah and Butler, 1996; Austin et al., 1999). Ovulation of persistent follicles and subsequent fertilization of senescent ova (Mihm et al., 1994; Revah and Butler, 1996) was associated with lower pregnancy rates due to increased embryonic death (Ahmad et al., 1995). In contrast, when using CIDR inserts for synchronization of the return to estrus, inserts are administered at about $14 \mathrm{~d}$ after $\mathrm{AI}$ and removed $7 \mathrm{~d}$ later ( $21 \mathrm{~d}$ after $\mathrm{AI})$ without administration of $\mathrm{PGF}_{2 \alpha}$. This treatment regimen is expected to minimize "extended" estrous cycles in nonpregnant cows that spontaneously regress their corpus luteum during the administration period, thus minimizing both the development of persistent follicles and the resulting reduction in CR in cows inseminated at estrus following insert removal.

The objectives of this study were to evaluate the effectiveness and safety of the CIDR insert (1.38 g of progesterone) when used to synchronize the return to estrus of previously inseminated lactating dairy cows with unknown pregnancy status.

\section{MATERIALS AND METHODS}

This study was conducted at eight commercial dairies (locations) using a common protocol. Data were pooled across all locations for statistical analyses. Dairies (n) were located in MI (1), NY (2), FL (1), IL (1), and CA (3) and ranged in size from 1100 to 5600 cows at the time the study was initiated. Daily milk production for these herds ranged from 27.3 to $38.2 \mathrm{~kg}$ per cow. The animal phase of the study was initiated in March 2001 and was completed in November 2001.

Cows were subjected to normal management practices within each location. Cows were fed a TMR using feed components typical to those used in the geographical region. Rations were balanced to meet National Research Council (1989) requirements for the appropriate stage of lactation. Cows enrolled into the study were housed with cows not enrolled in order to not disrupt the normal management of the cows at each dairy and to aid in the detection of estrus. Cows were housed in open-sided barns that were equipped with curtains in the northern locations. All barns had a centralized feed alley equipped with feed bunk head lockups for restraint of cows. Locations in warmer environments were equipped with water misters and fans to cool cows when restrained at the feed bunks. Physical examinations, AI, and insertion and removal of CIDR inserts were conducted when cows were restrained at the feed bunks.

A schedule of activities is presented in Table 1. Commercial grade, lactating Holstein dairy cows either not previously inseminated following the voluntary waiting period or found not pregnant, by palpation per rectum or return to estrus, to a previous insemination were eligible for participation in this study. Cows $\geq$ 40 or $\leq 150 \mathrm{~d}$ after calving, with four or fewer inseminations in the current lactation were eligible for enrollment. All cows were subjected to a physical examination by a veterinarian within $25 \mathrm{~d}$ before enrollment. Those cows with clinical observations of respiratory disease, mastitis, metritis, pyometra, uterine adhesions, or chronic or severe lameness during the physical examination were not eligible for enrollment. During the physical examination BCS were assessed using a 1 to 5 scale (Wildman et al., 1982). Cows with BCS $<2$ and $>4$ were not eligible for inclusion in the study.

All cows meeting the enrollment criteria were administered $25 \mathrm{mg}$ of $\mathrm{PGF}_{2 \alpha}(5 \mathrm{ml}$; LUTALYSE Sterile Solution; Pharmacia \& Upjohn Company, Kalamazoo, 
Table 1. Schedule of events for the animal phase of the study.

\begin{tabular}{lll}
\hline $\begin{array}{l}\text { Experimental } \\
\text { day }\end{array}$ & $\begin{array}{l}\text { Days post } \\
\text { preenrollment } \\
\text { insemination }\end{array}$ & Activity \\
\hline-25 to 0 & & $\begin{array}{l}\text { Physical examination, identification as eligible for enrollment. } \\
\text { Day of prostaglandin } \mathrm{F}_{2 \alpha} \text { injection } \\
\text { Observation for estrus, artificial insemination (AI) and enrollment of inseminated } \\
\text { cows (preenrollment estrus-synchronization period). } \\
\text { CIDR }\end{array}$ \\
$2,3,4$ & & $\begin{array}{l}\text { insert administration and daily general health observations begin. } \\
\text { Daily observation for estrus and AI begins. }\end{array}$ \\
17 & 13 to 15 & $\begin{array}{l}\text { CIDR insert removal and mucous score determination. } \\
\text { Last day of detection of estrus, AI, and general health observations. }\end{array}$ \\
21 & 17 to 19 & $\begin{array}{l}\text { Pregnancy diagnosis for pre-enrollment AI. } \\
\text { Pregnancy diagnosis for animals inseminated during the re-synchronization period }\end{array}$ \\
29 & 20 to 22 &
\end{tabular}

${ }^{1} \mathrm{CIDR}=$ intravaginal progesterone insert.

MI) to synchronize their preenrollment estrus. The day of $\mathrm{PGF}_{2 \alpha}$ injection was defined as experimental day 0 (EXD 0). Cows were observed for estrus using location-specific procedures. At six locations, estrusdetection procedures consisted of once daily reading of tail chalk. At one location, tail chalk and visual observation were utilized. At the remaining location, tail chalk, visual observations and pedometers were used to detect estrous activity. Those cows detected in estrus following $\mathrm{PGF}_{2 \alpha}$ administration were artificially inseminated using location-specific procedures. At six locations, cows were inseminated once daily after the morning observation for estrus. At the remaining two locations, cows were inseminated twice daily; however, at these two locations the majority of inseminations were conducted in the morning. Cows that were inseminated on EXD 2, 3, or 4 (preenrollment estrus-synchronization period) were enrolled in the study and were assigned randomly in blocks, based on time of availability, to each of two treatment groups: 1) control, no further treatment, or 2) administration of a CIDR insert on EXD 17 (d $14 \pm 1$ after insemination). A designated treatment administrator dispensed CIDR inserts using a specifically designed applicator. At most locations, the treatment administrator was a farm employee responsible for detecting estrus and AI. Inserts were removed $7 \mathrm{~d}$ after administration (EXD 24, $21 \pm 1 \mathrm{~d}$ after insemination). Cows eligible for enrollment and enrolled cows were managed in weekly cohorts with injection of $\mathrm{PGF}_{2 \alpha}$, administration of CIDR inserts and removal of CIDR inserts each performed weekly on a recurring day for each activity.

Cows were observed for signs of estrus using sitespecific procedures on EXD 21 to 29 (18 \pm 1 to $26 \pm 1$ $\mathrm{d}$ after the preenrollment insemination), herein referred to as the resynchronization-of-estrus period. Cows observed in estrus were inseminated artificially following location-specific procedures. In addition, each cow was observed for clinical signs of disease once daily during the $7 \mathrm{~d}$ that CIDR inserts were in place and for the $5 \mathrm{~d}$ after insert removal. At the time of removal, the vaginal mucus adhering to each insert was observed by the treatment administrator and a mucous score was recorded; score $1=$ no mucus observed, $2=$ clear mucus, $3=$ cloudy mucus, $4=$ yellow mucus, and $5=$ red or brown mucus. This scoring system was used to assess, indirectly, the severity of any localized vaginal irritation.

Every enrolled cow had their pregnancy status determined by a licensed veterinarian via either rectal palpation or ultrasonography at a target of 35 to 45 $\mathrm{d}$ after the preenrollment insemination. Cows inseminated during the resynchronization-of-estrus period had their pregnancy status determined a second time at 35 to $45 \mathrm{~d}$ after that insemination.

Initially, each location was asked to enroll 220 cows. This number was selected on the basis of eight locations and to have $80 \%$ power to be able to detect a difference of 10 percentage points $(\alpha=0.1)$ in CR to inseminations during resynchronization-of-estrus period. However, as the study progressed, one location was not able to enroll 220 cows in a timely manner; thus, the five locations that had not completed animal enrollment were asked to increase the number of animals enrolled to between 240 to 250 cows.

\section{Statistical Analyses}

Effectiveness. The primary variable for statistical analyses for effectiveness was synchronization of the return to estrus (SR). For the CIDR insert treatment group this was defined, a priori, as the percentage of cows in estrus on EXD 25, 26, and 27; the $3 \mathrm{~d}$ after CIDR insert removal. For the control group, the return-to-estrus data were pooled across all locations, and the three consecutive days with the greatest number of cows in estrus were selected. In this case EXD 
25,26 , and 27 again were selected. Cows subsequently determined to be pregnant to AI during the preenrollment period and cows that never had pregnancy status determined to AI during the preenrollment period (pregnancy status unknown) were not included in these analyses; they did not contribute to the denominator for the calculation of SR. Therefore, synchronization of return to estrus was calculated as:

$\mathrm{SR}=[\#$ of cows in estrus $\div$ (\# of cows enrolled - \# of cows pregnant to preenrollment AI - \# of cows with pregnancy status unknown)] $\times 100$.

After evaluation of the pattern in the return to estrus, the following additional ancillary analysis was conducted. The number of control cows in estrus during the entire 9-d resynchronization-of-estrus observation period (EXD 21 to 29) was compared to the number of cows receiving CIDR inserts that were in estrus on EXD 26 to 29.

Time to estrus during the resynchronization-of-estrus period also was evaluated as an ancillary variable for effectiveness. Time to estrus was defined for the CIDR insert group as the number of days from removal of the insert on EXD 24 to observation of estrus and for the control group as the number of days starting on EXD 21 to the observed estrus.

Fertility. Conception rate and pregnancy rate $(\mathbf{P R})$ were used to assess treatment effects on fertility, as measurements of safety of the use of CIDR inserts. Pregnancy rate of all cows inseminated during the preenrollment period (all cows enrolled; $\mathbf{P} \mathbf{R}_{\text {pre }}$ ) was considered a primary analysis. This evaluated the potential effect of CIDR insert administration in cows that had conceived to the preenrollment inseminations (effect of CIDR insert administered to pregnant animals).

$\mathrm{PR}_{\text {pre }}$ was calculated as:

$$
\begin{aligned}
& \mathrm{PR}_{\text {pre }}=[\# \text { pregnant } \div(\# \text { enrolled }-\# \text { with } \\
& \text { pregnancy status unknown })] \times 100 .
\end{aligned}
$$

Conception rate and $\mathrm{PR}$ for all inseminations conducted during the 9-d resynchronization-of-estrus period (EXD 21 to 29) also were considered to be primary analyses. These variables evaluated the potential effect of CIDR insert administration on CR and PR to inseminations immediately following insert removal.

Conception rate was calculated as:

$$
\begin{gathered}
\mathrm{CR}=[\# \text { pregnant to inseminations during the } \\
\text { resynchronization-of-estrus period } \div \\
\text { (\# inseminated during the resynchronization-of- }
\end{gathered}
$$

estrus period - \# with pregnancy

status unknown)] $\times 100$.

Pregnancy rate was calculated as:

$\mathrm{PR}=[\#$ pregnant to inseminations during the resynchronization-of-estrus period $\div$ (\# enrolled

- \# pregnant to the preenrollment inseminations

- \# with pregnancy status unknown)] $\times 100$.

The preplanned ancillary variable evaluating fertility was the cumulative PR to inseminations during the preenrollment synchronization period $\left(\mathrm{PR}_{\text {pre }} ; 3 \mathrm{~d}\right.$ of $\mathrm{AI}$ ) and the resynchronization-of-estrus period ( $9 \mathrm{~d}$ of AI), combined. As identified above, after evaluation of the pattern in the return to estrus the following analyses were conducted: Conception rate and $\mathrm{PR}$ to inseminations over the 9-d resynchronization-of-estrus period for control compared to inseminations over $4 \mathrm{~d}$ (EXD 26 to 29) for the CIDR insert treatment.

Statistical model and analyses. The study design was a randomized block conducted at multiple locations. Mixed logistic regression analyses were used for both primary and ancillary analyses of SR, PR, and CR. The model included terms for the random effects of location, block within location (when reasonable), and location $\times$ treatment interaction. Treatment was included in the model as a fixed effect. Covariates considered for inclusion in the models included parity (first lactation vs. all other lactations combined), days postpartum at time of enrollment, BCS, days from $\mathrm{PGF}_{2 \alpha}$ administration to $\mathrm{AI}$ during the preenrollment estrus-synchronization period and month of enrollment (grouped as March/April, May/June, and July/ August/September). Covariates were retained in the model when either their main effect or their interaction with treatment was significant $(\alpha \leq 0.10)$. The SAS GLIMMIX macro was used for these analyses. The test of significance for the primary variable of SR (efficacy) was made using a one-sided test $(\alpha=0.05)$ of the hypothesis that treatment with a CIDR insert was greater than control. For the primary measurements of fertility, as measurements of target animal safety, a one-sided test $(\alpha=0.10)$ of the hypothesis that CIDR insert was less than control was used, consistent with requirements of the Center for Veterinary Medicine, US Food and Drug Administration. For the ancillary measurements of cumulative PR and CR over EXD 21 to 29 for control and EXD 26 to 29 for CIDR insert, a two-sided test $(\alpha=0.05)$ of the hypothesis that CIDR insert is different than control was used. Estrous detection and PR over EXD 21 to 29 for control and EXD 26 to 29 for CIDR insert were tested with a 
one-sided test ( $\alpha=0.05$ ) of the hypothesis that CIDR insert is greater than control.

For time to estrus, proportional hazards regression analysis was used (SAS Proc PHREG). Location was treated as a strata variable to adjust for the effect of location.

\section{RESULTS}

During the course of the study, 1893 cows were enrolled into the study (945 control and 948 CIDR insert); however, 1754 were included in the analysis (867 control and 887 CIDR insert). Cows not included in the statistical analyses were excluded due primarily to documented deviations to the study protocol such as; enrolled without a physical examination; observed in estrus and inseminated during the interval of EXD 6 to 19; culled, subjected to euthanasia, or died before pregnancy diagnosis; the first 43 cows enrolled at one site without controls being assigned; not meeting enrollment criteria for days postpartum.

Over all locations, 97.3\% (863/887) of the CIDR inserts administered were retained for the scheduled 7$\mathrm{d}$ administration period. Across the eight locations retention rates ranged from a low of $91.9 \%$ at one location to a high of $100 \%$ at two locations. Cows that lost their inserts during the 7-d administration period were retained in the study and were included in appropriate statistical analyses.

Mucous scores were obtained for each of the 863 cows that retained the CIDR insert for $7 \mathrm{~d}$ : score $1=$ $7 \% ; 2=26 \% ; 3=38 \% ; 4=27 \%$; and $5=2 \%$. Thus, a majority of cows (65\%) had scores 3 or 4 , suggestive of localized irritation, and only $2 \%$ of cows had a score of 5, suggestive of severe irritation and, potentially, vaginitis. A mal-odor also was noted on study records in certain cases. Irritation was not apparent by external signs, such as vaginal discharge. Adverse health events related to administration of CIDR inserts were reported in only two cows; one cow had a vaginal discharge that was reported one time and was not evident when examined by the investigator the following day and one cow had extensive vaginal discharge, containing pus, on EXD 23. On the following day, at the time of scheduled removal, the CIDR insert was found to have turned sideways within the vaginal canal; no medical intervention was administered; however, vaginal discharge continued for more than 2 wk before resolving.

Health events noted during the daily observations and throughout the study were those that would be expected for cows during this stage of lactation. Based on the number of observations, mastitis was the primary medical event recorded (65 control and 66 CIDR

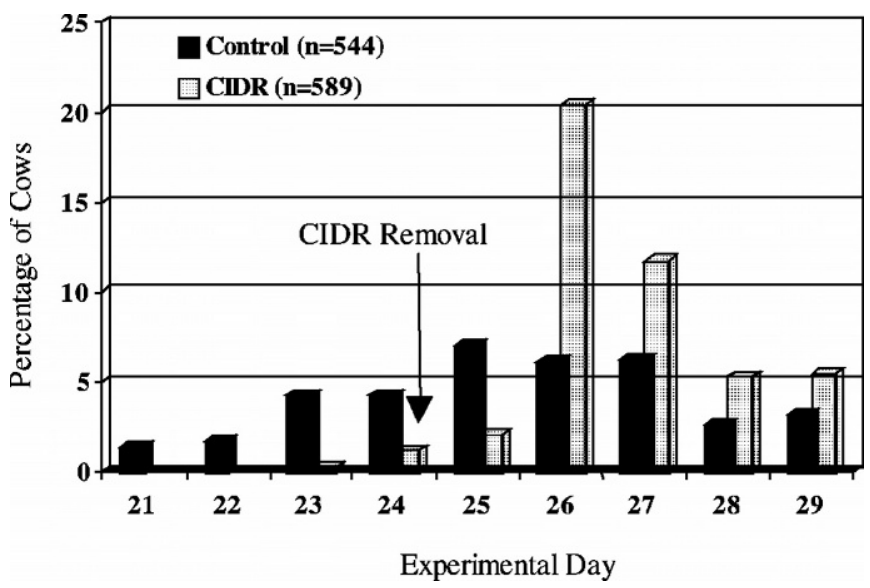

Figure 1. Percentage of cows that returned to estrus (of those potentially able to return to estrus) on each day of the resynchronization-of-estrus period (experimental d 21 to 29). Cows were inseminated on experimental $\mathrm{d} 2,3$, and 4 and intravaginal progesterone insert (CIDR) inserts were administrated on experimental d 17.

insert cows) followed by problems with feet and legs (five control and four CIDR insert cows), the respiratory tract (three control and four CIDR insert cows), and the gastrointestinal tract (one control and nine CIDR insert cows). Health observations in the gastrointestinal tract included bloat, left-displaced abomasum, peritonitis, stomach ulcer, abomasitits, indigestion, and lymphosarcoma. Visual appraisal of these data revealed no indication of treatment group trends; therefore, health events were not analyzed further.

Only one cow of the 887 cows administered CIDR inserts included in the statistical analyses data set was observed in estrus during the 7-d administration period; on EXD 23, $1 \mathrm{~d}$ before scheduled removal. One additional cow was observed in estrus and was inseminated on EXD 19 (2 d after insert administration); this cow was censored and not included in the analyses dataset. An additional seven cows were observed in estrus on the day of insert removal (EXD 24), however, three of these seven cows had lost their inserts before scheduled removal.

Figure 1 presents the percentage of cows observed in estrus of those able to return to estrus (not pregnant to AI during the preenrollment period) during the resynchronization-of-estrus period (EXD 21 to 29). A treatment effect $(P=0.001$; Table 2$)$ was detected for SR. In the CIDR insert treatment, $34.1 \%$ of those cows potentially able to return to estrus were observed in estrus during EXD 25, 26, and 27 compared with $19.3 \%$ of cows in the control observed in estrus during these $3 \mathrm{~d}$. Two covariates, neither significant as main effects, were found to have a significant interaction with treatment, BCS and day from $\mathrm{PGF}_{2 \alpha}$ to preenroll- 
Table 2. Percent (n) and significance levels for overall treatment effects.

\begin{tabular}{|c|c|c|c|c|c|}
\hline \multirow[b]{2}{*}{ Variable } & \multicolumn{2}{|c|}{ Control } & \multicolumn{2}{|c|}{ CIDR insert } & \multirow[b]{2}{*}{$P$-Value 8} \\
\hline & Mean (n) & $\operatorname{Min}-\operatorname{Max}^{7}$ & Mean (n) & Min - Max & \\
\hline $\mathrm{SR}^{1}$ & $19.3 \%(544)$ & $12.2 \%-32.2 \%$ & $34.1 \%(589)$ & $22.4 \%-40.7 \%$ & 0.001 \\
\hline $\mathrm{PR}_{\text {pre }}{ }^{2}$ & $36.7 \%(863)$ & $26.7 \%-47.3 \%$ & $32.7 \%(881)$ & $21.1 \%-46.0 \%$ & 0.044 \\
\hline $\mathrm{CR}^{3}$ & $30.9 \%(194)$ & $14.3 \%-45.0 \%$ & $26.7 \%(266)$ & $13.2 \%-46.9 \%$ & 0.271 \\
\hline $\mathrm{PR}^{4}$ & $11.1 \%(540)$ & $4.1 \%-19.7 \%$ & $12.2 \%(583)$ & $6.3 \%-25.0 \%$ & 0.650 \\
\hline Cum $P^{5}$ & $44.0 \%$ & $32.7 \%-57.7 \%$ & $41.2 \%$ & $27.8 \%-59.8 \%$ & 0.277 \\
\hline SR 9/4 & $36.4 \%(544)$ & $28.6 \%-49.3 \%$ & $42.8 \%(589)$ & $30.3 \%-50.7 \%$ & 0.030 \\
\hline CR $9 / 4^{6}$ & $30.9 \%(194)$ & $14.3 \%-45.0 \%$ & $27.1 \%(247)$ & $14.3 \%-46.9 \%$ & 0.587 \\
\hline $\operatorname{PR~} 9 / 4^{6}$ & $11.1 \%(540)$ & $4.1 \%-19.7 \%$ & $11.5 \%(583)$ & $5.3 \%-21.7 \%$ & 0.903 \\
\hline \multicolumn{6}{|c|}{ onization of the return to estrus; $\%$ of cows in estrus on experimental days (EXD) 25} \\
\hline \\
\hline \multicolumn{6}{|c|}{$\begin{array}{l}\left.{ }^{2} \mathrm{PR} \text { pre }=\text { Pregnancy rate to insemination during the pre-enrollment period (EXD } 2 \text { to } 4\right) \\
\left.{ }^{3} \mathrm{CR}=\text { Conception rate to insemination during the resynchronization period (EXD } 21 \text { to } 29\right) \text {. }\end{array}$} \\
\hline \multicolumn{6}{|c|}{${ }^{4} \mathrm{PR}=$ Pregnancy rate to insemination during the resynchronization period (EXD 21 to 29). } \\
\hline \multicolumn{6}{|c|}{$\begin{array}{l}{ }^{5} \text { Cum } \mathrm{PR}=\text { Pregnancy rate to insemination during the pre-enrollment plus resynchronization periods. } \\
{ }^{6} \mathrm{SR} 9 / 4, \mathrm{CR} 9 / 4, \mathrm{PR} 9 / 4=\text { Synchronization of return to estrus, conception rate, and pregnancy rate using }\end{array}$} \\
\hline \multirow{2}{*}{\multicolumn{6}{|c|}{$\begin{array}{l}9 \mathrm{~d} \text { of observation for Control (EXD } 21 \text { to } 29 \text { ) and } 4 \mathrm{~d} \text { of observation for CIDR-treated cows (EXD } 26 \text { to } 29 \text { ). } \\
\text { Calculation and analyses of these variables were not planned in the study design. }\end{array}$}} \\
\hline & & & & & \\
\hline \multicolumn{6}{|c|}{${ }^{7}$ Min - Max are the minimum and maximum percents among the eight trial locations. } \\
\hline
\end{tabular}

ment insemination (Table 3). The differences among treatment means indicated that the CIDR insert group always had a higher SR, but the magnitude of the difference between treatment means varied with BCS (Table 3) and day of insemination (Table 3). These results support the conclusion that the use of the CIDR insert synchronized the return to estrus more precisely than that observed in the control cows.

The median interval for the time to estrus during the resynchronization-of-estrus period was EXD 27 (the fifth day after EXD 21) for the control group and EXD 27 (the third day after EXD 24) for the CIDR insert group $(P<0.001)$. More cows $(P=0.031)$ potentially able to return to estrus were in estrus in the CIDR insert treatment over EXD 26 to 29 than were observed in estrus over the entire 9-d resynchronization-of-estrus period in the control (SR 9/4, Table 2). Day of insemination during the preenrollment period was a significant covariate for this analysis (Table 3.)

A treatment effect $(P=0.044)$ was detected in $P R_{\text {pre }}$; control cows had a slightly higher PR than cows in the CIDR insert treatment (36.7 vs. $32.7 \%$, respectively; Table 2). This implies that the administration of a CIDR insert to previously inseminated cows resulted in a loss of pregnancy in an estimated $11 \%$ of pregnant animals [100(36.7 - 32.7)/36.7]. The final $\mathrm{PR}_{\text {pre }}$ model contained three covariates; parity, BCS score and day from PGF injection to insemination. None of these covariates indicated a significant interaction with treatment (Table 3). Primiparous cows had higher $\mathrm{PR}_{\text {pre }}$ than multiparous cows (Table 3$), \mathrm{PR}_{\text {pre }}$ increased with increasing BCS (Table 3), and $\mathrm{PR}_{\text {pre }}$ increased with increasing day of insemination after PGF injec- tion (Table 3). Among cows administered CIDR inserts, an association was detected between mucous score and $\mathrm{PR}_{\text {pre }}(P=0.01$; Pearson chi-square test). The $P R_{\text {pre }}$ of cows with vaginal discharge scores of 1 , 2 , and 3 , combined, was $35 \%$ compared to $26 \%$ for cows with vaginal discharge scores of 4 and 5 , combined.

No difference was detected ( $P=0.271$; one-sided; Table 2) in CR to all inseminations during the 9-d resynchronization-of-estrus period; therefore, it cannot be concluded that use of CIDR reduced CR to AI immediately following insert removal. Parity was included as a significant covariate $(P=0.055$; Table 3$)$ with primiparous cows having a higher conception rate than multiparous cows; this effect was consistent across treatment. Among cows administered CIDR inserts the CR was $30 \%$ in cows with vaginal discharge scores of 1,2 , and 3 combined and was $19 \%$ in cows with vaginal discharge scores of 4 and 5 combined ( $P$ $=0.075$; Pearson's chi-square).

No difference was detected $(P=0.650$; one-sided; Table 2) in PR to inseminations during the 9-d resynchronization-of-estrus period; therefore it cannot be concluded that use of CIDR insert reduced PR to inseminations immediately following insert removal. Parity was included as a significant covariate with primiparous cows having a higher pregnancy rate than multiparous cows; this effect was consistent across treatments (Table 3).

No difference was detected in cumulative PR to inseminations during the preenrollment period combined with the 9-d period of resynchronization of estrus combined ( $P=0.277$; two-sided; Cum PR, Table 2 ); thus, it cannot be concluded that use of CIDR insert 
Table 3. Significant covariate effects that were included in the final statistical models and their probability levels.

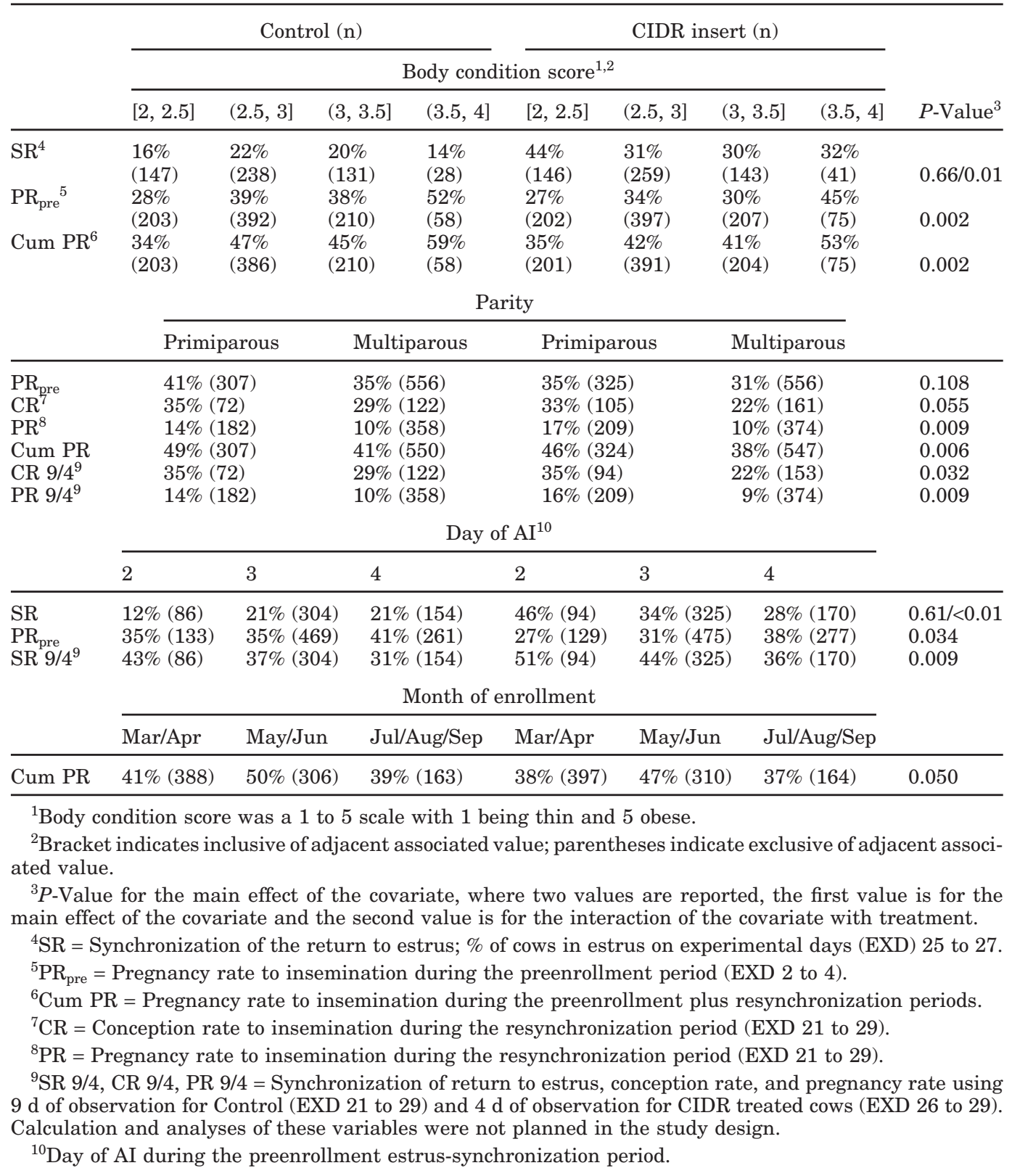

produced a different cumulative $\mathrm{PR}$ relative to that of the controls.

No treatment effects were detected $(P>0.5)$ in either $\mathrm{CR}$ or PR to inseminations conducted during the 4-d interval of EXD 26 to 29 for cows receiving CIDRinserts vs. inseminations during the 9 -d period of resynchronization of estrus for control cows (CR 9/4 and PR 9/4, Table 2). These observations demonstrate the practical utility of the use of CIDR inserts for synchronization of the returns to estrus; labor for estrous detection and $\mathrm{AI}$ of cows returning to estrus following a previous insemination can be concentrated over a 3 or $4 \mathrm{~d}$ following CIDR insert removal rather than over $9 \mathrm{~d}$ if CIDR inserts were not used.

\section{DISCUSSION}

Retention rate of the progesterone intravaginal CIDR inserts over the 7-d administration period across all locations was $97.3 \%$. This retention is interpreted to be acceptable.

The CIDR insert effectively inhibited the expression of estrus during the 7-d insertion period as evidenced 
by the minimal number of cows detected in estrus during the administration period $(\mathrm{n}=2 ; 0.2 \%)$.

Administration of CIDR inserts resulted in a more precise return to estrus (Figure 1) and a greater SR than those observed in control cows; $34.1 \%$ of cows administered the CIDR insert were observed in estrus over the 3-d period of synchronization of returns to estrus in contrast to $19.3 \%$ of control cows over the same $3 \mathrm{~d}$. This observation is consistent with results reported by others (Macmillan and Peterson, 1993; Macmillan and Burke, 1996; Van Cleeff et al., 1996).

The significant interaction between treatment and BCS (covariate) on SR may be explained, in part, to a high SR response of cows with low BCS to the administration of CIDR inserts (Table 3). The greatest difference between treatment groups in SR was seen in the cows with BCS of 2.0 to 2.5 . Only $16 \%$ of control cows with BCS of 2.0 to 2.5 were detected in estrus on EXD 25 to 27, whereas $44 \%$ of cows administered CIDR inserts were detected in estrus during those $3 \mathrm{~d}$. This may be a result of a beneficial effect of short-term exposure to supplemental progesterone on subsequent estrous behavior or on the continuation of estrous cyclicity of cows with low BCS.

The significant interaction between treatment and day of AI following $\mathrm{PGF}_{2 \alpha}$ administration on $\mathrm{SR}$ is not easily explained (Table 3 ). It is possible that this is only a consequence of the days selected for inclusion in the calculation of SR; EXD 25, 26, and 27. In the analysis for SR using $9 \mathrm{~d}$ for controls and $4 \mathrm{~d}$ for cows receiving CIDR inserts, interaction between treatment and day of $\mathrm{AI}$ following $\mathrm{PGF}_{2 \alpha}$ administration was not detected (Table 3).

For synchronization of return to estrus, the data identified that EXD 26,27 , and 28 provided a greater percentage of cows in estrus for the CIDR insert group rather than EXD 25, 26, and 27 (Figure 1). Also, a 4$\mathrm{d}$ window (EXD 26 to 29) may be more appropriate than the 3-d window that was defined in the protocol. By selection of this shorter period, 23.2\% of CIDR treated cows observed in estrus were not included in the analyses of synchronization of return to estrus. The pattern in the return to estrus of cows previously inseminated and administered CIDR inserts is consistent with that reported by Macmillan and Peterson (1993); when CIDR inserts were removed $21 \mathrm{~d}$ post$\mathrm{AI}$, of cows detected in estrus $2.2 \%$ were observed on 20 to $22 \mathrm{~d}$ post-AI and $67.4 \%$ were detected at 23 or $24 \mathrm{~d}$ post-AI. Comparable percentages for the same days postinsemination for the study reported herein are 7.4 and $69.5 \%$, respectively.

The pattern in the return to estrus of control cows also warrants further discussion. In general, estrous cycles of 18 to $24 \mathrm{~d}$ are considered normal, with $21 \mathrm{~d}$ considered the average duration. Of the control cows detected in estrus in this study, numerically more were observed in estrus on EXD $25(38 / 198=19.2 \%$; $22 \pm 1 \mathrm{~d}$ after previous estrus and insemination) than other days; there were 23 (11.6\%), 23 (11.6\%), 33 (16.7\%), and $34(17.2 \%)$ cows observed on EXD 23, 24, 26 , and 27, respectively. Thus, the duration of the estrous cycle was skewed to intervals longer than 21 d. An additional $15.7 \%$ of control cows and $23.2 \%$ of cows administered a CIDR insert were observed in estrus on EXD 28 and 29 combined ( $25 \pm 1$ and $26 \pm$ $1 \mathrm{~d}$ post AI). These data are consistent with estrous cycles of $23.9 \pm 0.8 \mathrm{~d}$ for noninseminated, Holstein cows $(n=14)$ reported by Sartori et al $(2000)$. However, embryonic loss after d 16 of gestation cannot be precluded as a possible cause for this extension in length of the estrous cycle in some cows (Northey and French, 1980; Thatcher and Hansen, 1992; Van Cleeff et al., 1991). These observations raise the question: Is the average duration of the estrous cycle of lactating Holstein cows in the United States longer than the generally accepted average of $21 \mathrm{~d}$ ?

Only $19.3 \%$ of control cows, potentially available to express estrus, returned to estrus on EXD 25, 26, and 27 . Furthermore, the pattern of returns to estrus following CIDR insert removal was spread over more days than anticipated. These observations led to the conduct of additional ancillary statistical analyses. When the $9 \mathrm{~d}$ of estrus observations were included (EXD 21 to 29), only $36 \%$ of the control cows were observed in estrus. In contrast, $34.1 \%$ of cows administered CIDR inserts were observed over $3 \mathrm{~d}$ (EXD 25 to 27 ), and $42.8 \%$ over the $4 \mathrm{~d}$ with the highest incidence of cows observed in estrus (EXD 26 to 29). It is assumed that detection of estrus is improved when cohorts of cows are in estrus, simultaneously; therefore, the large number of CIDR-treated cows in estrus over $4 \mathrm{~d}$ may have aided the detection of estrus in cows in the control group thus inflating the detected estrous response in the control group. These observations are consistent with the general low rate of estrous detection in commercial herds in the United States. Low detection of estrus may be due to shortened duration of estrous behavior in modern dairy cows, to housing conditions not conducive to estrous display (concrete floors), insufficient time spent detecting estrus or reliance on secondary observations (tail chalk) for detection of estrus (Lucy, 2001). These observations, however, identify the potential value of use of the CIDR insert to synchronize the return to estrus. More cows treated with CIDR inserts were observed in estrus during a 4-d interval following insert removal than the number of control cows observed in estrus over the entire 9-d period of resynchroniza- 
tion of estrus. Thus, CIDR inserts administered to synchronize the return to estrus provide an additional tool to dairy producers to better manage labor; labor to conduct estrous detection and AI of cows can be intensified/concentrated over a 3 to $4 \mathrm{~d}$ interval following CIDR insert removal to enhance PR of cows returning to estrus.

Fertility levels of the herds in this study are within dairy industry norms. The percentage of cows pregnant to $\mathrm{AI}$ at 2,3 , and $4 \mathrm{~d}$ after an injection of $\mathrm{PGF}_{2 \alpha}$ were 36.7 and $32.7 \%$ in the control and CIDR treatment, respectively. In addition, $\mathrm{PR}_{\mathrm{pre}}, \mathrm{PR}$, and $\mathrm{CR}$ were higher in first-parity cows compared with second and greater parity cows. This provides evidence of the attention to management of first-parity cows within these locations.

The small (4.0\%), yet significant $(P=0.044)$, reduction in $\mathrm{PR}_{\text {pre }}$, detected in cows receiving CIDR inserts was not anticipated and is not consistent with results reported by others. In general, supplementation or stimulation of blood progesterone concentration in cattle, by various means, from 4 to $16 \mathrm{~d}$ after insemination have been reported to have either a beneficial or no effect on subsequent pregnancy rate (Thatcher and Hansen, 1992). A reduction in conception rate has been reported only when progesterone supplementation was provided on 1 to $7 \mathrm{~d}$ or 2 to $7 \mathrm{~d}$ after AI (Van Cleeff et al., 1991). The concept for increasing progesterone during early pregnancy is to stimulate development of the conceptus to improve long-term survival (Thatcher and Hansen, 1992). Administration of a progesterone releasing intravaginal device from 13 to $21 \mathrm{~d}$ after first service of lactating Holstein cows had no effect on first service conception rates (Stevenson and Mee, 1991). Administration of previously used CIDR inserts to lactating dairy cows from 13 to $20 \mathrm{~d}$ after fixed-time insemination did not have a detrimental effect on pregnancy rates to the preceding fixed-time insemination (El-Zarkouny et al., 2001). Macmillan and Peterson (1993) evaluated the use of CIDR B inserts to synchronize the return to estrus of previously inseminated lactating dairy cows and to provide supplemental progesterone during the diestrous period of the estrous cycle. In one study, 514 cows in seven herds were administered CIDR B inserts starting from 14 to $17 \mathrm{~d}$ after first insemination and inserts were removed $22 \mathrm{~d}$ after insemination. The control group consisted of 472 previously inseminated but otherwise untreated cows. Pregnancy rates to first insemination were 64 and $63.6 \%$ in the CIDR B insert treated and control cows, respectively. In another study, conducted in 11 herds, CIDR B inserts were administered to 493 cows starting 10 to $16 \mathrm{~d}$ after insemination and were removed $6 \mathrm{~d}$ later. Control cows $(n=628)$ were untreated. Pregnancy rates to first insemination were 64.3 and $66.6 \%$ in the cows administered CIDR B inserts and control cows, respectively. Van Cleeff et al. (1996) administered previously used CIDR B inserts to 112 dairy heifers at $17 \mathrm{~d}$ after insemination and removed them $21 \mathrm{~d}$ after insemination. Control heifers $(\mathrm{n}=138)$ were untreated following first insemination. Pregnancy rates to first insemination were 43.5 and $50.0 \%$ in the control and CIDR$\mathrm{B}$ treated heifers, respectively. In contrast, $\mathrm{Xu}$ and Burton (1999) reported a reduction in PR in dairy heifers administered previously used CIDR B inserts to synchronize the return to estrus. In that study, CIDR B inserts were administered $16 \mathrm{~d}$ after insemination and removed $21 \mathrm{~d}$ after insemination. However, with the design of their study, it is not possible to determine the cause of the reduced pregnancy rate. Heifers treated with CIDR B inserts had the previous estrus synchronized with $10 \mathrm{mg}$ of estradiol benzoate administered at the time of insert administration. The administration period for CIDR B inserts was $10 \mathrm{~d}$ with $\mathrm{PGF}_{2 \alpha}$ administered on d 6 . Heifers in this group were fixed-time inseminated at $50 \mathrm{~h}$ after insert removal. In contrast, heifers in the control group did not have the previous estrus synchronized and were inseminated following detection of estrus. Therefore, the reduced PR in heifers treated with the CIDR B insert may have been in response to: 1) an inappropriate timing of the fixed-time AI; 2) administration of $\mathrm{PGF}_{2 \alpha} 4 \mathrm{~d}$ before insert removal; 3) the extended administration period ( $10 \mathrm{~d}$ ) of the CIDR B inserts; or 4) use of CIDR B inserts to synchronize the return to estrus.

The associations detected between higher mucous scores and lower PR to inseminations during the preenrollment period $\left(\mathrm{PR}_{\text {pre }}\right)$ and to lower $\mathrm{CR}$ to $\mathrm{AI}$ during the resynchronization-of-estrus period are interesting, but cannot be used to positively identify a cause/effect relationship. Nonetheless, this observation strongly supports a recommendation to use sanitary methods to minimize the potential for contamination of inserts or applicators during administration of CIDR inserts. Such methods should reduce the incidence of vaginal irritation and vaginitis and may help to minimize any reduction in pregnancy rates.

The observation of no difference detected between treatment groups in $\mathrm{CR}$ to $\mathrm{AI}$ during the period of resynchronization of estrus is consistent with the hypothesis that the adminstration of a CIDR insert does not result in the formation of persistent follicles in those cows that spontaneously regress their CL during the administration period. This observation is consistent with the observations of others (Macmillan and Peterson, 1994; Van Cleeff et al., 1996). 


\section{CONCLUSIONS}

When using proper administration techniques, retention of CIDR inserts in lactating, Holstein dairy cows was excellent. Observation of mucus at the time of CIDR insert removal suggests that the insert caused mild localized intravaginal irritation in a majority of cows and potential vaginitis in less than $2 \%$ of treated cows. The lack of observations of vaginal discharge during daily general health observations during the administration period and on the $5 \mathrm{~d}$ following insert removal supports that this irritation and potential vaginitis was self-limited. No other general health observations or medical events were noted that would suggest a detrimental effect on the health of cows that could be attributed to use of CIDR inserts as described in this study.

Administration of CIDR inserts to lactating dairy cows on d $14 \pm 1$ after insemination with removal $7 \mathrm{~d}$ later ( $21 \pm 1 \mathrm{~d}$ after insemination) effectively synchronized returns to estrus. More cows were observed in estrus 2 to $5 \mathrm{~d}$ after removal of the CIDR insert than were seen in control cows over the 9 -d of expected return to estrus ( $18 \pm 1$ to $26 \pm 1 \mathrm{~d}$ after previous insemination). These observations demonstrate the practical utility of the use of CIDR inserts for synchronization of the return to estrus.

Cows receiving CIDR inserts had a slightly lower PR than that observed in the control cows to inseminations conducted immediately pretreatment (32.7 vs. $36.7 \% ; P=0.044)$. This observation implies that the administration of CIDR inserts to previously inseminated cows resulted in a loss of pregnancy in an estimated $11 \%$ of pregnant cows. This observation is not consistent with previously published data that reported no negative effects of CIDR inserts on PR to inseminations immediately preceding CIDR insert administration.

No difference was detected between treatment groups for CR or PR to AI conducted during the 9-d period of resynchronization of estrus (i.e., following CIDR insert removal). No difference was detected between treatment groups in cumulative PR to AI. Thus it cannot be concluded that the administration of CIDR inserts had a detrimental effect on the fertility of inseminations conducted at estrus immediately following insert removal.

The presence of severe vaginal irritation and/or vaginitis in cows administered CIDR inserts was associated with reduced $\mathrm{PR}$ to $\mathrm{AI}$ during the preenrollment period and with reduced CR to AI during the period of resynchronization of estrus. Therefore, it is recommended to use proper sanitation procedures when ad- ministering CIDR inserts to minimize contamination of CIDR inserts.

The results of this study support the conclusion that, when properly used, CIDR inserts are safe and effective for synchronization of returns to estrus of previously inseminated lactating dairy cows with unknown pregnancy status. Such use of the CIDR insert results in cows returning to estrus over fewer days following a previous insemination, thus allowing dairy producers to intensify or concentrate labor needed to conduct estrous detection and AI of cows returning to estrus.

\section{ACKNOWLEDGMENTS}

The authors would like to acknowledge the investigators and support staff at all eight trial locations for their perseverance to complete this protocol: Paul Busman, D. V. M., Darrel Kesler, Ph. D, Steve Carlson, D. V. M., Jose Santos, D. V. M., Ph. D, Phillip Jardon, D. V. M., Arthur Sherman, D. V. M., Anthony Wiseley, D. V. M., and James P. Garlough. Their efforts to conduct this study in accordance with the protocol are gratefully acknowledged.

\section{REFERENCES}

Ahmad, N., F. N. Schrick, R. L. Butcher, and E. K. Inskeep. 1995. Effect of persistent follicles on early embryonic losses in beef cows. Biol. Reprod. 52:1129-1135.

Austin, E. J., M. Mihm, M. P. Ryan, D. H. Williams, and J. F. Roche. 1999. Effect of duration of dominance of the ovulatory follicle on the onset of estrus and fertility in heifers. J. Anim. Sci. $77: 2219-2226$.

Cooperative Regional Research Project, NE-161. 1996. Relationship of fertility to patterns of ovarian follicular development and associated hormonal profiles in dairy cows and heifers. J. Anim. Sci. 74:1943-1952.

El-Zarkouny, S. Z., J. A. Cartmill, A. M. Richardson, M. A. MedinaBritos, and J. S. Stevenson. 2001. Presynchronization of estrous cycles in lactating dairy cows with Ovsynch + CIDR and resynchronization of repeat estrus using the CIDR. J. Dairy Sci. 84(Suppl. 1):249. (Abstr.)

Lucy, M. C. 2001. Reproduction loss in high-producing dairy cattle: Where will it end? J. Dairy Sci. 84:1277-1293.

Macmillan, K. L., and A. J. Peterson. 1993. A new intravaginal progesterone releasing device for cattle (CIDR-B) for estrous synchronization, increasing pregnancy rates and the treatment of post-partum anoestrus. Anim. Reprod. Sci. 33:1-25.

Macmillan, K. L., and C. R. Burke. 1996. Effects of estrous control on reproductive efficiency. Anim. Reprod. Sci. 42:307-320.

Mihm, M., N. Curran, P. Hytteel, M. P. Boland, and J. F. Roche. 1994. Resumption of meiosis in cattle oocytes from preovulatory follicles with a short and long duration of dominance. J. Reprod. Fertil. Abstr. Series 13:14.

National Research Council. 1989. Nutritional Requirements of Dairy Cattle. 6th rev. ed. Natl. Acad. Sci., Washington DC.

Northey, D. L., and L. R. French. 1980. Effect of embryo removal and intrauterine infusion of embryonic homogenates on the life span of the bovine corpus luteum. Theriogenology 50:298-302.

Ogle, C. R. 1999. Design, development and optimization of veterinary intravaginal controlled release drug delivery systems. Ph.D. Thesis, University of Waikato, Hamilton, NZ. 
Revah, I., and W. R. Butler. 1996. Prolonged dominance of follicles and reduced viability of bovine oocytes, J. Reprod. Fertil. 106:39-47.

Ryan, D. P., S. Snijders, H. Yaakub, and K. J. O'Farrell. 1995. An evaluation of estrous synchronization programs in reproductive management of dairy herds. J. Anim. Sci. 73:3687-3695.

Ryan, D. P., J. A. Galvin, and K. J. O'Farrell. 1999. Comparison of oestrous synchronization regimens for lactating dairy cows. Anim. Reprod. Sci. 56:153-168.

Sartori, R., J. Haughian, G. J. M. Rosa, R. D. Shaver, and M. C. Wiltbank. 2000. Differences between lactating cows and nulliparous heifers in follicular dynamics, luteal growth, and serum steroid concentrations. J. Dairy Sci. 83(Suppl. 1):212. (Abstr.)

Savio, J. D., W. W. Thatcher, L. Badinga, R. L. de la Sota, and D. Wolfenson. 1993a. Regulation of dominant follicle turnover during the oestrus cycle in cows. J. Reprod. Fertil. 97:197-203.

Savio, J. D., W. W. Thatcher, G. R. Morris, K. Entwistle, M. Drost, and M. R. Mattiacci. 1993b. Effects of induction of low plasma progesterone concentrations with a progesterone intravaginal device on follicular turnover and fertility in cattle. J. Reprod. Fertil. 98:77-84.

Stevenson, J. S., and M. O. Mee. 1991. Pregnancy rates of Holstein cows after postinsemination treatment with a progesteronereleasing intravaginal device. J. Anim. Sci. 74:3849-3856.
Thatcher, W. W., and P. J. Hansen. 1992. Systems to alter embryo survival. Pages 164-174 in Large Dairy Herd Management. H. H. Van Horn and C. J. Wilcox, ed. American Dairy Science Association, Champaign, IL.

Van Cleeff, J., M. Drost, and W. W. Thatcher. 1991. Effects of postinsemination progesterone supplementation on fertility and subsequent estrous responses of dairy heifers. Theriogenology $36: 795-807$.

Van Cleeff, J., K. L. Macmillan, M. Drost, M. C. Lucy, and W. W. Thatcher. 1996. Effects of administering progesterone at selected intervals after insemination of synchronized heifers on pregnancy rates and resynchronization of returns to service. Theriogenology 46:1117-1130.

Wildman, E. E., G. M. Jones, P. E. Wagner, R. L. Boman, H. F. Troutt, Jr., and T. N. Lesch. 1982. A dairy cow body condition scoring system and its relationship to selected production characteristics. J. Dairy Sci. 65:495-501.

Xu, Z. Z., L. J. Burton, and K. L. Macmillan. 1996. Reproductive performance of lactating dairy cows following oestrus synchronization with progesterone, oestradiol and prostaglandin. N.Z. Vet. J. 44:99-104.

Xu, Z. Z., and L. J. Burton. 1999. Reproductive performance of dairy heifers after estrus synchronization and fixed-time artificial insemination. J. Dairy Sci. 82:910-917. 